\title{
Announcement: Changes to the Table of Contents of Physical Review E
}

We are pleased to announce several changes to the table of contents of Physical Review E.

As of January 1, 2013, the journal will no longer consist of two parts. Most of our readers access the journal online, and this unification should streamline the online table of contents. Because of its size the printed journal will still be split, but the division is no longer based on subject matter, solely on the number of pages. The subtitles for the two individual parts will be retired.

The journal now includes thirteen sections, with the implementation of the following changes. First, the section on equilibrium and linear transport properties of fluids, which has become quite small, has been removed, and in the future these papers will be published in suitable sections of the journal. Second, the sections on colloids and complex fluids have been merged as these two naturally fit together. Third, the section on chaos and pattern formation has been renamed "Nonlinear Dynamics and Chaos" which better reflects its coverage.

We stress that there is no change in the scope of Physical Review E, and that we are implementing these changes to improve the organization of the journal.

Published 2 January 2013

DOI: 10.1103/PhysRevE.87.010001

PACS number(s): 01.10.Cr, 01.30.-y 